\title{
Pure laparoscopic liver resection for giant liver hemangioma with extrahepatic growth based on preoperative 3-dimensional simulation: A case report
}

\author{
Yuichiro Okumura', Takehiro Noda ${ }^{1}$, Hidetoshi Eguchi ${ }^{1 *}$ D, Takehiko Hanaki ${ }^{1}$, Yoshifumi Iwagami ${ }^{1}$, Hirofumi Akita ${ }^{1}$, \\ Tadafumi Asaoka', Kunihito Gotoh', Shogo Kobayashi ${ }^{1}$, Koji Umeshita $^{2}$, Masaki Mori ${ }^{1,3}$ and Yuichiro Doki ${ }^{1}$
}

\begin{abstract}
Background: Performing laparoscopic liver resection for giant hemangiomas is challenging, and careful preoperative planning is essential. Controlling intraoperative bleeding and handling surgical instruments within a limited workspace is necessary.

Case presentation: In the present case, the patient was a 38-year-old woman diagnosed with a 16-cm giant liver hemangioma in segment 5/6, with extrahepatic growth. Preoperative three-dimensional simulations for port placement and the laparoscopic view from the left upper abdomen were performed to complete the pure laparoscopic liver resection. The laparoscopic resection was then safely performed on the same way.

Conclusions: Pure laparoscopic resection could be applied to giant hemangiomas with extrahepatic growth, and the preoperative three-dimensional simulation of port placement and the laparoscopic view might be helpful when the intraabdominal workplace is restricted.
\end{abstract}

Keywords: Giant hemangioma, Laparoscopic liver resection, 3D simulation

\section{Introduction}

Cavernous hemangiomas are the most common type of benign liver tumors, and the majority of cavernous hemangiomas are incidentally detected by imaging studies performed for other reasons [1]. A recent national survey reported that liver hemangiomas measuring $>10 \mathrm{~cm}$ would be candidates for surgery in patients with symptoms [2]. Compared with open liver resection, laparoscopic liver resection (LLR) has many advantages including shorter hospital stay, less blood loss, and earlier postoperative recovery [3]. The current indication for LLRs is a solitary tumor with a $\leq 5-\mathrm{cm}$ diameter [4]. LLR for giant hemangiomas $>10 \mathrm{~cm}$ is challenging due to insufficient space for manipulation and bleeding risk [2, 3]. Here, we present a case with a symptomatic giant hemangioma $16 \mathrm{~cm}$ in

\footnotetext{
* Correspondence: heguchi@gesurg.med.osaka-u.ac.jp

1 Department of Gastroenterological Surgery, Graduate School of Medicine,

Osaka University, 2-2, Yamadaoka E-2, Suita, Osaka 565-0871, Japan

Full list of author information is available at the end of the article
}

diameter that was successfully resected by LLR after utilizing three-dimensional (3D) simulation technology to preoperatively plan port placement and the laparoscopic view.

\section{Case presentation}

A young woman was referred to our hospital for a hepatic mass, which presented with epigastric pain. The patient had no notable past medical history. She did not smoke or abuse alcohol. Her physical examination revealed slight epigastric tenderness. Contrast-enhanced computed tomography $(\mathrm{CT})$ demonstrated a 9 -cm mass in segments $5 / 6$ of the liver, with extrahepatic growth. The radiological findings were compatible with cavernous hemangioma. Because the patient's symptoms soon disappeared, she decided to receive surveillance. For 6 years after her first visit to the hospital, the hemangioma grew slowly. However, during the seventh year, the tumor grew from $13 \mathrm{~cm}$ to $16 \mathrm{~cm}$, and surgery was indicated. 
Preoperative laboratory tests revealed that the patient's hemoglobin level was slightly decreased at $116 \mathrm{~g} / \mathrm{L}$, but the liver function tests, platelet counts, and coagulation factors were all within the normal ranges. Figure $1 \mathrm{a}$ and $\mathrm{b}$ show the CT images of the giant hemangioma originating from segments 5/6, with a maximum diameter of $16 \mathrm{~cm}$. The preoperative 3D simulation was conducted using SYNAPSE VINCENT (Fujifilm Medical, Tokyo, Japan). The laparoscopic view and parenchymal cutline for the pure laparoscopic approach were simulated by inserting trocars mainly on the left upper abdomen (Fig. 2a, b). The positional relation between the hemangioma and the vasculature was also simulated (Additional file 1: Video 1). Transcatheter arterial embolization (TAE) to A5 as a main feeder was performed using Embosphere, which was diluted 100 times, on the day before surgery.

During laparoscopy, carbon dioxide was used for peritoneal insufflation, and the abdominal pressure was maintained between 8 and $12 \mathrm{mmHg}$. Observed from the umbilical laparoscopic view, the giant hemangioma occupied the whole space (Fig. 3a). All ports were placed as in the preoperative simulation. The hepato-duodenum ligament was encircled by approaching the lesser omentum. An umbilical tape was guided to the omental foramen, using snake retractor (Additional file 1: Video 1). Pringle maneuver was applied during the transection. The straight resection line was confirmed using intraoperative ultrasound, and hepatic transection was done by laparosonic coagulating shears (Ethicon Endo-Surgery, Cincinnati, OH, USA), an ultrasonic surgical aspirator (CUSA; Cavitron Lasersonic Corp., Stamford, CT, USA), and a bipolar clamp coagulation system (the VIO 300D; ERBE Elektromedizin, Tübingen, Germany) (Fig. 3b, c). The hemangioma was then extracted using a plastic bag through the slightly enlarged umbilical trocar incision (Fig. 4). A drainage tube was placed adjacent to the resection margin (Fig. 3d). The operative time was $288 \mathrm{~min}$, and total blood loss was 200
$\mathrm{mL}$ (Additional file 1: Video 1). Transfusion was not required. The resected tumor was $16 \times 16 \times 9 \mathrm{~cm}$ in diameter and weighed $980 \mathrm{~g}$. Histopathological findings were compatible with cavernous hemangioma. The patient had an uneventful recovery.

\section{Discussion}

Cavernous liver hemangiomas are typically observed in adults aged 30-50 years, and occur three to five times more frequently in women [1]. Giant hemangiomas may be complicated by hematologic or coagulation system abnormalities known as Kasabach-Merritt syndrome [2, 5], and some patients complain of abdominal symptoms. The operative indication for liver hemangiomas is determined by their size, growth rate, and presence of symptoms. Prophylactic resection in asymptomatic patients solely due to size is regarded as inadequate [6]. Sakamoto et al. conducted the national survey of Japan and reported that about half of patients with 10-15-cm hemangiomas had no clinical symptoms and could be candidates for careful observation [2]. Our patient received observation, with imaging done every 6 months, but the tumor rapidly increased in size and exceeded $15 \mathrm{~cm}$, fulfilling the indication for hepatic resection.

LLR was first introduced in 1992 and is increasingly used due to new technologies and devices [7]. Compared to open liver resection, LLR has several advantages including decreased blood loss, shorter hospital stay, and improved cosmetic results. The acceptable indication for LLR according to the Louisville Statement is a solitary liver tumor $\leq 5 \mathrm{~cm}$ [4]. Recently, using LLR for large hemangiomas has been reported $[8,9]$. The difficulty with using LLR for giant hemangiomas depends on tumor size, tumor location, growth type, and adhesion area between the hemangioma and liver.

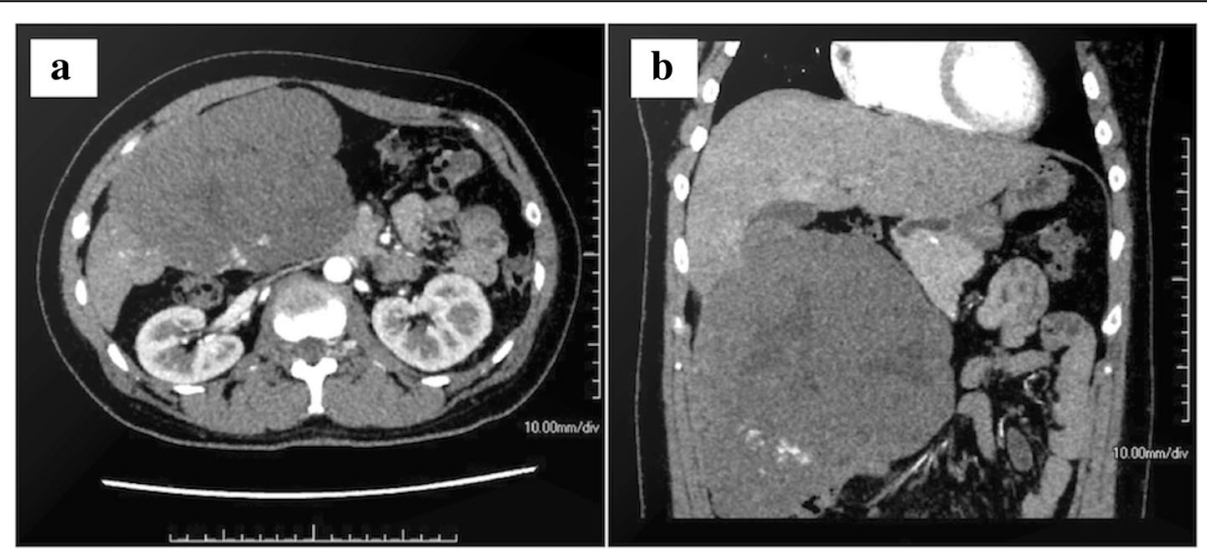

Fig. 1 Contrast-enhanced computerized tomography showing a giant liver hemangioma originating from segments 5/6, with extrahepatic growth. a Axial section. b Coronal section 


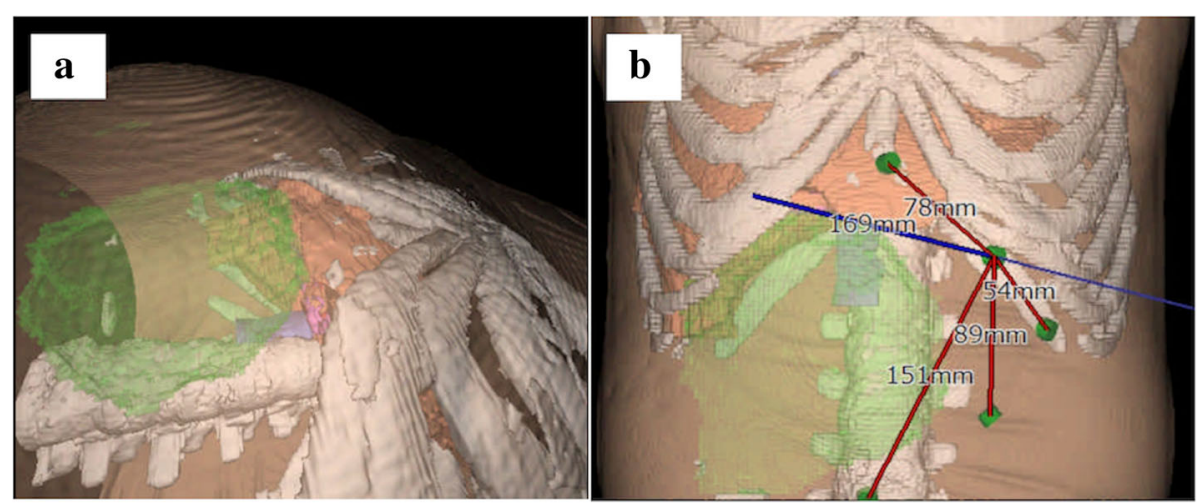

Fig. 2 Preoperative three-dimensional simulation images. a Reconstructed laparoscopic view from the left upper abdomen. b Port placement and the minimal straight parenchymal cutline were simulated

Because hemangiomas have high vascularity and a soft texture, they have the potential to bleed easily. Controlling the inflow into the resection area is essential, and preoperative TAE is an effective modality to reduce operative bleeding related with the hepatic arterial supply $[8,10-13]$. Zhang et al also reported that infrahepatic inferior vena cava clamping with the Pringle maneuver was safe and effective for controlling bleeding [9]. In our case, preoperative TAE and the Pringle maneuver were applied. The operative time $(288 \mathrm{~min})$ and blood loss $(200 \mathrm{~mL}$ ) were normal and comparable to that previously reported [9], and TAE would be somewhat helpful to lessen bleeding.

Preoperative simulation using 3D image reconstruction may enhance surgical planning and navigation during LLR [14]. It can measure the remnant liver volume and visualize hepatic anatomy and tumor localization. In LLR for giant hemangiomas, handling several laparoscopic instruments within a limited workspace is key to completing the operation. Our patient's giant hemangioma showed extrahepatic growth, and the intraabdominal workspace was severely restricted. The simulated laparoscopic view from the left upper abdomen was optimal for safely handling the various surgical instruments, and $3 \mathrm{D}$ simulation was very informative and helpful for port placement. Though, the change of positional relationship among the organs and costal bones under pneumoperitoneum could not be considered in this simulation tool. The definitive decision of port placement should be conducted intraoperatively. This is the first report in which preoperative 3D simulation of port site placement, and the laparoscopic view

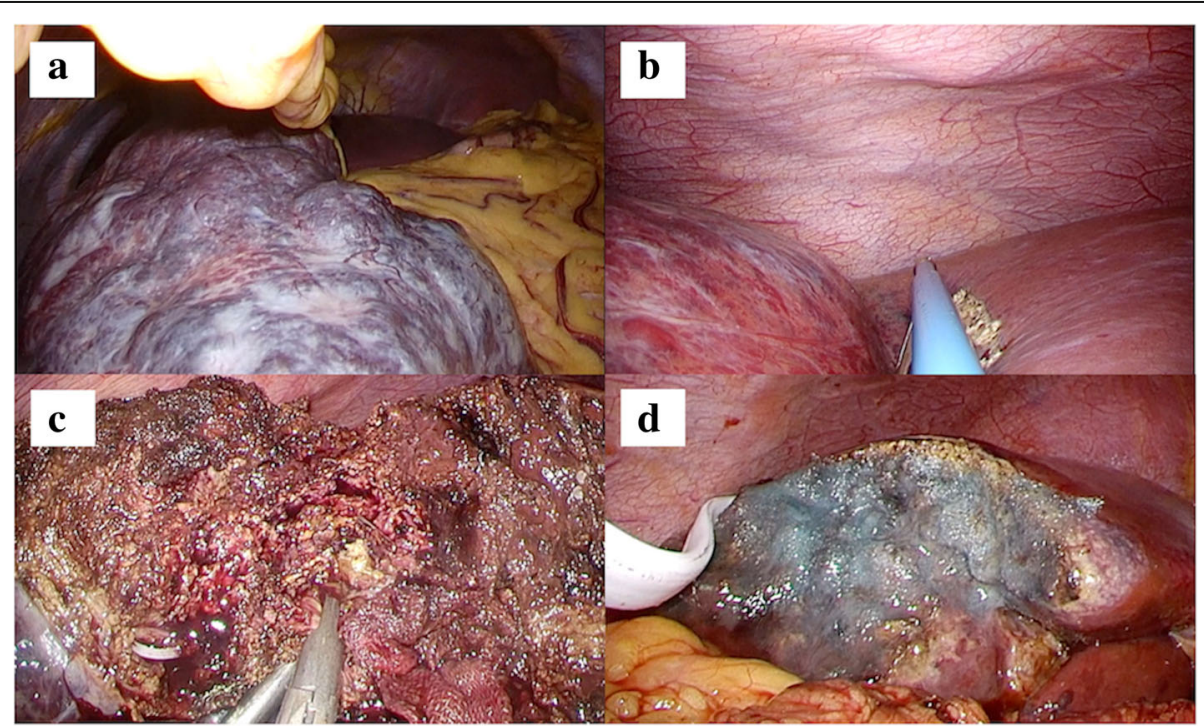

Fig. 3 a The giant hemangioma occupying the entire space of the right upper abdomen was observed from the umbilical laparoscopic view. $\mathbf{b}$ A straight transection cutline was marked on the liver surface from the left upper laparoscopic view. $\mathbf{c}$ Hepatic transection. $\mathbf{d}$ After the resection, a drainage tube was placed adjacent to the resection margin 


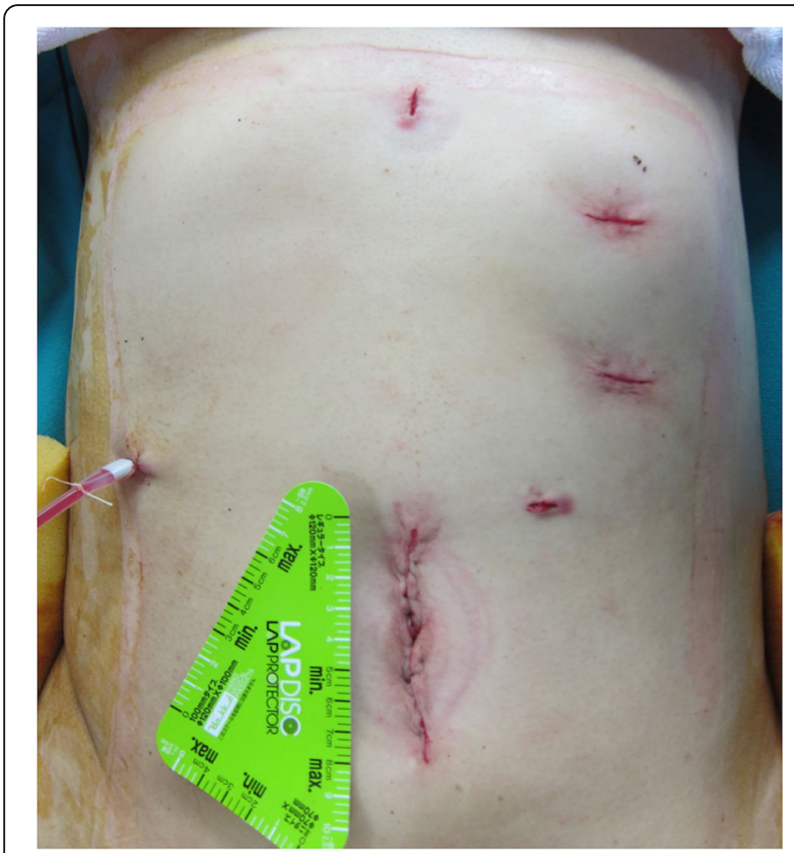

Fig. 4 Postoperative abdominal incision. The umbilical trocar incision was slightly extended and used to extract the specimen

was utilized in LLR for giant liver hemangioma. The parenchymal transection plane image, the laparoscopic view, and the port placement were simulated preoperatively, and the operation was successfully completed the same way it was simulated.

In conclusion, our report suggests that pure laparoscopic resection could be safely applied to resect giant liver hemangiomas with extrahepatic growth. The preoperative 3D simulation of the port placement and the laparoscopic view might be helpful when intraabdominal workspace is restricted.

\section{Additional file}

Additional file 1: Video 1 . The shortly edited movie of the threedimensional simulation view from the left upper abdomen and the whole intraoperative video. (MP4 $19570 \mathrm{~kb}$ )

\section{Abbreviations}

3D: Three-dimensional; CT: Computed tomography; LLR: Laparoscopic liver resections; TAE: Transcatheter arterial embolization

\section{Acknowledgements}

Not applicable.

\section{Funding}

The authors of this manuscript have received no grants or financial support concerning this study.

\section{Availability of data and materials}

Data sharing is not applicable to this article as no datasets were generated or analyzed during the current study.

\section{Authors' contributions}

YO, TN, and HE conceived and designed the study. YO, TN, TI, HA, TA, KG, and SK acquired the data. YO, KU, MM, and YD drafted the manuscript. All authors read and approved the final manuscript.

\section{Consent for publication}

Written informed consent was obtained from the patient involved in this publication and accompanying images.

\section{Competing interests}

The authors declare that they have no competing interest.

\section{Publisher's Note}

Springer Nature remains neutral with regard to jurisdictional claims in published maps and institutional affiliations.

\section{Author details}

${ }^{1}$ Department of Gastroenterological Surgery, Graduate School of Medicine, Osaka University, 2-2, Yamadaoka E-2, Suita, Osaka 565-0871, Japan. ²Division of Health Science, Graduate School of Medicine, Osaka University, Osaka, Japan. ${ }^{3}$ Department of Surgery and Science, Graduate School of Medical Sciences, Kyushu University, Fukuoka, Japan.

Received: 30 December 2018 Accepted: 18 March 2019 Published online: 01 April 2019

\section{References}

1. Erdogan D, Busch OR, van Delden OM, Bennink RJ, ten Kate FJ, Gouma DJ, et al. Management of liver hemangiomas according to size and symptoms. J Gastroenterol Hepatol. 2007;22:1953-8.

2. Sakamoto Y, Kokudo N, Watadani T, Shibahara J, Yamamoto M, Yamaue H. Proposal of size-based surgical indication criteria for liver hemangioma based on a nationwide survey in Japan. J Hepatobiliary Pancreat Sci. 2017; 24:417-25.

3. Wakabayashi G, Ikeda T, Otsuka Y, Nitta H, Cho A, Kaneko H. General gastroenterological surgery 3: liver. Asian J Endosc Surg. 2015;8:365-73.

4. Buell JF, Cherqui D, Geller DA, O'Rourke N, lannitti D, Dagher I, et al. The international position on laparoscopic liver surgery: the Louisville Statement, 2008. Ann Surg. 2009;250:825-30.

5. Liu X, Yang Z, Tan H, Liu L, Xu L, Sun Y, et al. Characteristics and operative treatment of extremely giant liver hemangioma $>20 \mathrm{~cm}$. Surgery. 2017;161: 1514-24.

6. Dong J, Zhang M, Chen JQ, Ma F, Wang HH, Lv Y. Tumor size is not a criterion for resection during the management of giant hemangioma of the liver. Eur J Gastroenterol Hepatol. 2015;27:686-91.

7. Chanwat R. Useful maneuvers for precise laparoscopic liver resection. Asia J Endosc Surg. 2018;11:93-103.

8. Kim IS, Kwon CHD. Feasibility of laparoscopic liver resection for giant hemangioma of greater than $6 \mathrm{~cm}$ in diameter. Korean J Hepatobiliary Pancreat Surg. 2014;18:118.

9. Z Zhang W, Wang J, Li C, Zhang Z, Dirie NI, Dong H, et al. Infrahepatic inferior vena cava clamping with Pringle maneuvers for laparoscopic extracapsular enucleation of giant liver hemangiomas. Surg Endosc. 2017;31:3628-36.

10. Topaloglu S, Oguz S, Kalayci O, Ozturk MH, Calik A, Dinc H, et al. Preoperative arterial embolization of large liver hemangiomas. Diagn Interv Radiol. 2015;21:222-8.

11. Furumaya A, van Rosmalen BV, Takkenberg RB, van Delden OM, Dejong CHC, Verheij J, et al. Transarterial (chemo-)embolization and lipiodolization for hepatic haemangioma. Cardiovasc Interv Radiol. 2019. [Epub ahead of print]

12. Lanthaler M, Freund $M$, Nehoda $H$. Laparoscopic resection of a giant liver hemangioma. J Laparoendosc Adv Surg Tech A. 2005;15:624-6.

13. Sun JH, Nie CH, Zhang YL, Zhou GH, Ai J, Zhou TY, et al. Transcatheter arterial embolization alone for giant hepatic hemangioma. PloS One. 2015; 10:e0135158.

14. Hallet J, Gayet B, Tsung A, Wakabayashi G, Pessaux P. Systematic review of the use of pre-operative simulation and navigation for hepatectomy: current status and future perspectives. J Hepatobiliary Pancreat Sci. 2015;22:353-62. 